\title{
Gamma-Ray Curvature Radiation from the Polar Regions of a Radio Pulsar with an Axisymmetric Magnetic Field
}

\author{
D. P. Barsukov, E. M. Kantor, and A. I. Tsygan \\ Ioffe Physicotechnical Institute, Russian Academy of Sciences, St. Petersburg, Russia \\ Received March 16, 2005; in final form, July 6, 2005
}

\begin{abstract}
The influence of an axisymmetric magnetic field on the intensity, spectrum, and shape of a pulse of gamma-ray curvature radiation from the polar regions of a radio pulsar is investigated. The pulsar is considered in a Goldreich-Julian model with a free-electron emission from the neutron-star surface. The influence on the curvature radiation of variations of both the curvature of the magnetic field lines and the electric field due to the nondipolarity of the magnetic field are investigated. The presence of even modest nondipolarity (less than 10\%) can lead to a sharp drop in the intensity of the gamma-ray curvature radiation, while the intensity of the $\mathrm{X}$-ray curvature radiation (photon energies $<100 \mathrm{keV}$ ) is affected only weakly.
\end{abstract}

PACS numbers : $97.60 . \mathrm{G}$

DOI: $10.1134 / \mathrm{S} 1063772906020090$

\section{INTRODUCTION}

The influence of nondipolarity of the magnetic field of a radio pulsar on the pulsar's X-ray and gammaray radiation is currently being actively studied. Interest in this question was called forth especially by the discovery in 1999 of a long-period radio pulsar [1]. The presence of a strong nondipolar component of the magnetic field at the surface of the neutron star was proposed in $[2,3]$ to explain the existence of this pulsar.

The X-ray and gamma-ray emission of radio pulsars with dipolar magnetic fields has been studied in detail in a number of works, such as [4,5] ("polar cap" model), [6, 7] ("outer gap" model), and [8] ("slot gap" model).

The first analysis taking into account nondipolarity of the magnetic field appeared in [9]. The effect of axially symmetric nondipolarity of the field on the electrodynamics of a pulsar operating in a regime with a free outflow of electrons from the surface of the neutron star was examined in [10]. In 1996, Pal'shin and Tsygan [11] considered the influence of nondipolarity of the magnetic field on the generation of electron-positron pairs and thermal X-ray radiation in the polar regions, taking into account the general relativistic effect of frame dragging. An expression for the electrostatic potential in a rotating reference frame for the case of an axially symmetric nondipolar magnetic field including general relativistic effects was obtained in 2000 [12]. In 2003, Kantor and Tsygan [13] obtained an expression for the electrostatic potential for an asymmetric magnetic field. The influence of nondipolarity of the magnetic field on the operation of a radio pulsar is studied in detail in [14, 15].

The current paper is a direct continuation of [11, $13,16]$. We consider here the effect on the characteristics of the gamma-ray curvature radiation of radio pulsars due to effects associated with curvature of the field lines, as well as the influence of nondipolarity of the magnetic field on the electrostatic potential.

We analyze a radio pulsar in a Goldreich-Julian model with a free-electron emission from the neutron-star surface. The pulsar diode is located at the surface of the neutron star (i.e., we consider a polar-cap model).

When calculating the nonthermal X-ray and gamma-ray radiation of the radio pulsar, we included only the curvature radiation of the ultrarelativistic primary electrons, and neglected radiation associated with inverse-Compton scattering of thermal photons from the neutron-star surface on the primary electrons and the radiation emitted by secondary electrons and positrons.

This approximation is quite justified if the surface of the neutron star is sufficiently cool. In the future, we plan to include the influence of inverse-Compton scattering on the gamma-ray radiation of the polar regions of a radio pulsar with a nondipolar magnetic field.

Here, we include only the absorption of photons in the magnetic field, associated with the creation of electron-positron pairs in an unbound state. We neglect all general-relativistic effects except frame 
dragging, which makes a significant contribution in the calculations of the electric field.

\section{THE NONDIPOLAR MAGNETIC FIELD}

We will describe the nondipolarity of the magnetic field using the model of [11] (see also [13]). Let the neutron star have a radius $a$ and a dipolar magnetic moment $\vec{m}$ (so that the field at its magnetic pole is $B_{0}=2 m / a^{3}$ ), with an additional dipole with magnetic moment $\mathbf{m}_{1}$ being located in the polar region of the neutron star at a depth of $a \Delta(\Delta<1)$ below the surface. The vector $\mathbf{m}_{1}$ is perpendicular to $\mathbf{m}$, lies in the $(\boldsymbol{\Omega}, \mathbf{m})$ plane, and is directed toward the rotational axis of the pulsar. The parameter $\Delta$ is taken to be 0.1 , which corresponds to the thickness of the neutron-star crust and does not lead to rapid decay of the additional dipole $\mathbf{m}_{1}$. We introduce the spherical coordinate system $(\eta=r / a, \theta, \phi)$ with $z \| \mathbf{m}$.

Then, disregarding for simplicity the curvature of space near the neutron star, we obtain in the smallangle approximation $\theta \ll 1$ the following expression for the total magnetic field $\mathbf{B}=\mathbf{B}_{0}+\mathbf{B}_{1}$ :

$$
\begin{gathered}
B_{\eta}=\frac{B_{0}}{\eta^{3}}, \quad B_{\theta}=\frac{B_{0}}{\eta^{3}}\left(\frac{\theta}{2}+\mu \cos \phi\right), \\
B_{\phi}=-\frac{B_{0}}{\eta^{3}} \mu \sin \phi
\end{gathered}
$$

where $\mu=\nu\left(\frac{\Delta \eta}{\eta-1+\Delta}\right)^{3}$ and $\nu=B_{1} / B_{0}$ is the ratio of the magnetic-field strengths $B_{1}$ and $B_{0}$ at the pole of the neutron $\operatorname{star}(\eta=1, \theta=0)$.

Let us consider the field lines in the plane of the vectors $\mathbf{m}_{1}, \mathbf{m}$. Their radius of curvature $\rho_{\mathrm{c}}$ is well described (when $\nu \leq 0.5, \Delta \approx 0.1$ ) by the approximate formula

$$
\rho_{\mathrm{c}}=2 \eta a\left\{\frac{3}{2} \theta+\mu\left[\left(\frac{3(1-\Delta)}{\eta-1+\Delta}\right)-\frac{1}{2}\right]\right\}^{-1} .
$$

\section{THE ELECTRIC FIELD}

We will use the results of Kantor and Tsygan [13], who constructed an electrodynamical model for a radio pulsar with a nondipolar magnetic field, taking into account general-relativistic frame dragging. The electrostatic potential $\Phi$ in the rotating coordinate system satisfies the Poisson equation [17, 18]:

$$
\operatorname{div}\left(\frac{1}{\alpha} \nabla \boldsymbol{\Phi}\right)=-4 \pi\left(\rho+\rho_{\text {eff }}\right),
$$

where $\quad \rho_{\text {eff }}=\frac{1}{4 \pi c} \operatorname{div}\left\{\frac{1}{\alpha}\left(1-\frac{k}{\eta^{3}}\right)[[\boldsymbol{\Omega} \times \mathbf{r}] \times \mathbf{B}]\right\}$ is the Goldreich-Julian density, $\rho$ is the electrical charge density associated with the particles flowing from the surface of the neutron star, $\alpha=\sqrt{1-\epsilon / \eta}$, $\epsilon=r_{\mathrm{g}} / a, r_{\mathrm{g}}=2 G M / c^{2}$ is the gravitational radius of the neutron star, $r$ is the radial coordinate in the Schwarzschild metric, $\eta=r / a, k=\epsilon \beta, \beta=I / I_{0}$ is the moment of inertia of the neutron star in units of $I_{0}=M a^{2}$, and $\boldsymbol{\Omega}$ is the angular-velocity vector for the neutron star's rotation.

Here and below, we will neglect for simplicity effects associated with the curvature of space near the neutron star, and assume that everywhere $\alpha \equiv 1$ (terms containing $k$ will be included). In particular, this means that we disregard the gravitational redshift of the photons.

Using (1) to find the magnetic field in the smallangle approximation $\theta \ll 1$, the expression for $\rho_{\text {eff }}$ takes the form [13]

$$
\begin{aligned}
\rho_{\text {eff }} & =\frac{\Omega B_{0}}{2 \pi c} \frac{1}{\eta^{3}}\left[\left(1-\frac{k}{\eta^{3}}\right) \cos \chi\right. \\
& \left.-\mu\left(1+\frac{1}{2} \frac{k}{\eta^{3}}\right) \sin \chi\right],
\end{aligned}
$$

where $\chi$ is the angle between $\boldsymbol{\Omega}$ and $\mathbf{m}$.

It is assumed that $\left.E_{\|}\right|_{\eta=1}=0$ at the surface of the neutron star (where $E_{\|}=(\mathbf{E B}) / B$ is the component of the electric field along $\mathbf{B})$, and that $\Phi=0$ at the walls of the pulsar tube. The presence of the upper diode plate (anode) at a height $z_{\mathrm{c}} \equiv \eta_{\mathrm{c}}-1$ is assumed; i.e., $\left.E_{\|}\right|_{\eta=\eta_{\mathrm{c}}}=0$.

The potential that is obtained under these conditions can be found in [13]. For a thin tube with a large height for the upper diode plate $z_{\mathrm{c}} \gg \theta_{\mathrm{s}}$ (where $\theta_{\mathrm{s}}$ is the transverse width of the tube in stellar radii), it takes the form

$$
\Phi=\frac{\Omega F}{2 \pi c}\left(1-\xi^{2}\right)\left[\left(1-\frac{k}{\eta^{3}}\right) f(\eta)-(1-k) f(1)\right],
$$

where $F=\pi\left(\theta_{\mathrm{s}} a\right)^{2} B$ is the magnetic flux in the tube,

$$
\begin{gathered}
f(\eta) \equiv\{\cos \chi \\
\left.-\mu\left[\left(1+\frac{1}{2} \frac{k}{\eta^{3}}\right) /\left(1-\frac{k}{\eta^{3}}\right)\right] \sin \chi\right\} / \sqrt{1+\mu^{2}}
\end{gathered}
$$

(when $k=0$, this quantity coincides with the cosine of the angle between $\boldsymbol{\Omega}$ and the value of the vector $\mathbf{B}$ on the tube axis).

For the case $z_{\mathrm{c}} \lesssim \theta_{\mathrm{s}}, z_{\mathrm{c}} \ll \Delta, \theta_{\mathrm{s}} \ll \Delta$ and with the angle between the magnetic field lines and the neutron-star surface much less than the maximum of 
the two quantities $\theta_{\mathrm{s}} / z_{\mathrm{c}}$ and $z_{\mathrm{c}} / \theta_{\mathrm{s}}$, our solution to (3) takes the form

$$
\begin{gathered}
\Phi=2 \Phi_{0} K_{1} \sum_{i=0}^{\infty} \frac{1}{\gamma_{i}^{2}}\left(z+\frac{F\left(z, \gamma_{i}\right)}{\gamma_{i}}\right) \frac{2}{k_{i} J_{1}\left(k_{i}\right)} J_{0}\left(k_{i} \xi\right), \\
\rho=\frac{\Omega B}{2 \pi c} A(\xi),
\end{gathered}
$$

where $\xi$ retains the meaning of the coordinate perpendicular to the tube axis ( $\xi=0$ corresponds to the tube axis and $\xi=1$ to the tube walls),

$$
\begin{aligned}
F(z, \gamma)= & -\frac{\left(1-e^{-\gamma z}\right)\left(1+e^{-\gamma\left(z_{\mathrm{c}}-z\right)}\right)}{1+e^{-\gamma z_{\mathrm{c}}}} \\
A(\xi)=- & K_{0}-K_{1} \sum_{i=0}^{\infty} \frac{1}{\gamma_{i}} \frac{\left(1-e^{-\gamma_{i} z_{\mathrm{c}}}\right)}{\left(1+e^{-\gamma_{i} z_{\mathrm{c}}}\right)} \\
& \times \frac{2}{k_{i} J_{1}\left(k_{i}\right)} J_{0}\left(k_{i} \xi\right),
\end{aligned}
$$

$\Phi_{0}=\frac{\Omega a}{c} B_{\mathrm{s}} a, B_{\mathrm{S}}$ is the magnetic field at the neutronstar surface at the center of the tube, $\gamma_{i}=k_{i} / \theta_{\mathrm{s}}, k_{i}$ is the root of the equation $J_{0}(k)=0$, and the expressions for the coefficients $K_{0}$ and $K_{1}$ have the form

$$
\begin{gathered}
K_{0}=(1-k) \frac{1}{\sqrt{1+\nu^{2}}} \cos \chi \\
-\left(1+\frac{1}{2} k\right) \frac{\nu}{\sqrt{1+\nu^{2}}} \sin \chi \\
K_{1}=\frac{3 \nu}{\left(1+\nu^{2}\right)^{3 / 2}} \frac{1-\Delta}{\Delta} \\
\times\left((1-k) \nu \cos \chi+\left(1+\frac{1}{2} k\right) \sin \chi\right) \\
+\frac{3 k}{\sqrt{1+\nu^{2}}}\left(\cos \chi+\frac{1}{2} \nu \sin \chi\right) .
\end{gathered}
$$

Expression (6) becomes equal to the potential obtained in [19] as $z_{\mathrm{c}} \rightarrow 0$ (which is also applicable when $\left.\theta_{\mathrm{s}} \approx \Delta\right)$,

$$
\Phi=\Phi_{0} K_{1}\left(\frac{z_{\mathrm{c}} z^{2}}{2}-\frac{z^{3}}{3}\right)
$$

but in contrast to the form for a dipolar field [18], the factor $K_{1} \sim 2-3$ is present in place of the factor $3 k \sim 0.5$ (when $\Delta \sim 0.1$ ). This shows that, when $z_{\mathrm{c}} \lesssim \theta_{\mathrm{s}}$, in the case of the "favorable" magnetic-field configuration we have chosen (other conditions being equal) [13], the potential due to the nondipolarity of the magnetic field can appreciably exceed the potential associated with frame dragging.

\section{GENERATION OF GAMMA-RAY EMISSION}

Everywhere in this section, we will use the following coordinate system. We consider a point $x_{0}$ on the central field line of the pulsar tube, and introduce the unit vector $\mathbf{e}_{B}$ tangent to this field line at the point $x_{0}$. We also introduce the vectors $\mathbf{e}_{1}$ and $\mathbf{e}_{2}$, such that the set $\left(\mathbf{e}_{B}, \mathbf{e}_{1}, \mathbf{e}_{2}\right)$ forms an orthonormal basis and $\mathbf{e}_{1}$ lies in the plane of the vectors $\mathbf{m}$ and $\boldsymbol{\Omega}$ and is directed toward the rotational axis of the pulsar.

The cross section of the pulsar tube made by the $\left(\mathbf{e}_{1}, \mathbf{e}_{2}\right)$ plane will be taken to be a circle with radius $a \theta_{\mathrm{s}}\left(x_{0}\right)$ with its center at the point $x_{0}$, where $a^{2} \theta_{\mathrm{s}}^{2}\left(x_{0}\right) B\left(x_{0}\right)=a^{2} \theta_{0}^{2} B_{0}$ (from the condition of flux conservation for the thin tube); $B\left(x_{0}\right)$ is the magnetic-field strength at the point $x_{0}$. We introduce the coordinates $\xi$ and $\phi$ for any point $x$ lying in the $\left(\mathbf{e}_{1}, \mathbf{e}_{2}\right)$ plane as follows:

$$
\mathbf{x}=\mathbf{x}_{0}+a \theta_{\mathrm{s}}\left(x_{0}\right)\left(\xi \cos \phi \mathbf{e}_{1}+\xi \sin \phi \mathbf{e}_{2}\right) .
$$

Thus, points with $\xi \leq 1$ lie inside the pulsar tube, while those with $\xi>1$ lie outside. The meaning of the coordinate $\eta=r / a$ remains the same.

Further, the coordinates $(\eta, \xi, \phi)$ introduced in this way will be used as the coordinates $(\eta, \xi, \phi)$ from Section 3 , and the line $\xi=$ const, $\phi=$ const will be taken to represent field lines of the magnetic field. We believe that this approximation is fully applicable in the case of thin tubes and $\theta \ll 1$; i.e., in the smallangle approximation.

The primary electrons flowing from the neutronstar surface are accelerated along magnetic field lines by the electric field (6).

We will disregard the drift across the magnetic field and transitions of electrons to upper Landau levels. The energy of the electrons at the point $x=(\eta, \xi, \phi)$ will be taken to be $\epsilon \equiv m c^{2} \Gamma=e \Phi+m c^{2}$, where $\Phi=\Phi(\eta, \xi, \phi)$ is the value of the potential (6) at the point $x, m$ is the mass of the electron, $c$ is the speed of light, and $e$ is the magnitude of the electron charge.

As they move along the curved magnetic field lines, the electrons emit gamma-ray curvature radiation with a power spectrum (in terms of numbers of particles) [20]

$$
\begin{gathered}
\frac{d N(x, \gamma)}{d t d \gamma}=\frac{\sqrt{3}}{2 \pi} \alpha_{\mathrm{F}}\left(\frac{c}{\rho_{\mathrm{c}}}\right) \beta \Gamma F\left(\frac{\gamma}{\gamma_{\mathrm{c}}}\right) \frac{1}{\gamma_{\mathrm{c}}}, \\
F(\xi)=\int_{\xi}^{+\infty} K_{5 / 3}(s) d s,
\end{gathered}
$$

where $\frac{d N}{d t d \gamma} d \gamma$ is the number of photons with energy $\gamma$ in the interval $d \gamma$ emitted by an electron at the point $x$ 


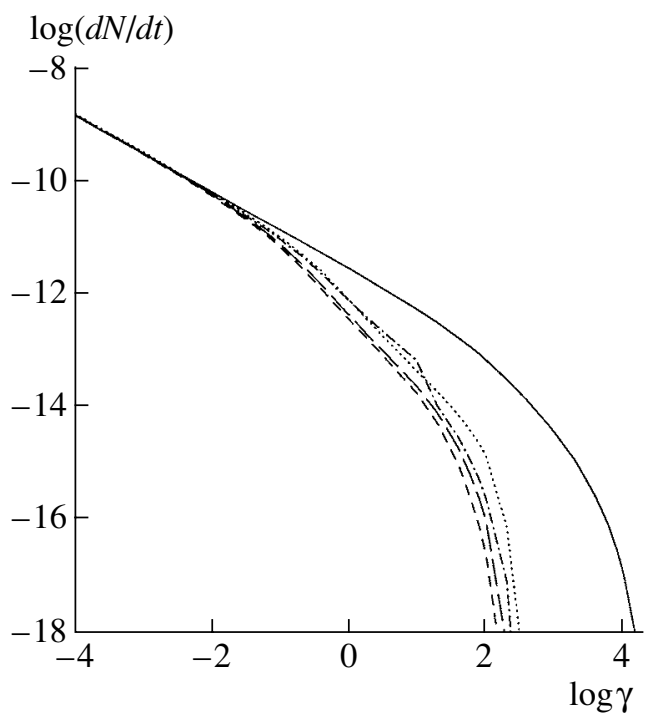

Fig. 1. Time-averaged spectrum of the curvature radiation of the pulsar tube for $B=0.05 B_{\mathrm{cr}}, P=0.2 \mathrm{~s}$, $\chi=10^{\circ}, k=0.15$, and $\Delta=0.1$; the angle between the line of sight and the rotational axis of the pulsar is $10^{\circ}$. The solid curve corresponds to a dipolar field $(\nu=0)$, and the dotted, long-dashed, short-dashed, and dash-dotted curves to nondipolar fields with $\nu=0.1$, $\nu=0.2, \nu=0.3$, and $\nu=0.5$, respectively. The intensity of the curvature radiation $d N / d t$ is measured in units of the flux received from a source located at a distance of $1 \mathrm{kpc}$ : photons $/ \mathrm{cm}^{2} \mathrm{~s} \mathrm{keV}$. The photon energy $\gamma$ is measured in units of $m c^{2}$.

per second, $\gamma=\frac{\hbar \omega}{m c^{2}}$ is the photon energy in units of $m c^{2}, \omega$ is the photon's frequency, $\gamma_{\mathrm{c}}=\frac{3}{2} \frac{\lambda_{\mathrm{c}}}{\rho_{\mathrm{c}}} \beta \Gamma^{3}$, $\Gamma$ is the Lorentz factor of an electron at the point $x$, $\beta=\frac{\sqrt{\Gamma^{2}-1}}{\Gamma}, \lambda_{\mathrm{c}}=\frac{\hbar}{m c}$ is the Compton wavelength of the electron, $\alpha_{\mathrm{F}}$ is the fine-structure constant, and $\rho_{\mathrm{c}}$ is the radius of curvature of the magnetic field lines at the point $x$ [calculated using (2)].

Since $\Gamma \gg 1$ in the region of interest to us, we will assume in our subsequent calculations that all photons emitted at the point $x$ have momenta directed along the magnetic-field vector $\mathbf{B}(x)$.

When calculating the intensity of the nonthermal $\mathrm{X}$-ray and gamma-ray emission of the pulsar tube, we will include only curvature radiation by the primary electrons inside the tube.

As the gamma-rays propagate, the angle $\Psi$ between the momentum of the gamma-rays and the magnetic-field vector $\mathbf{B}$ grows due to the curvature of the force lines. When $\gamma \sin \Psi>2$, it becomes possible for a gamma-ray to be absorbed in the magnetic field, accompanied by the creation of an electron-positron pair.

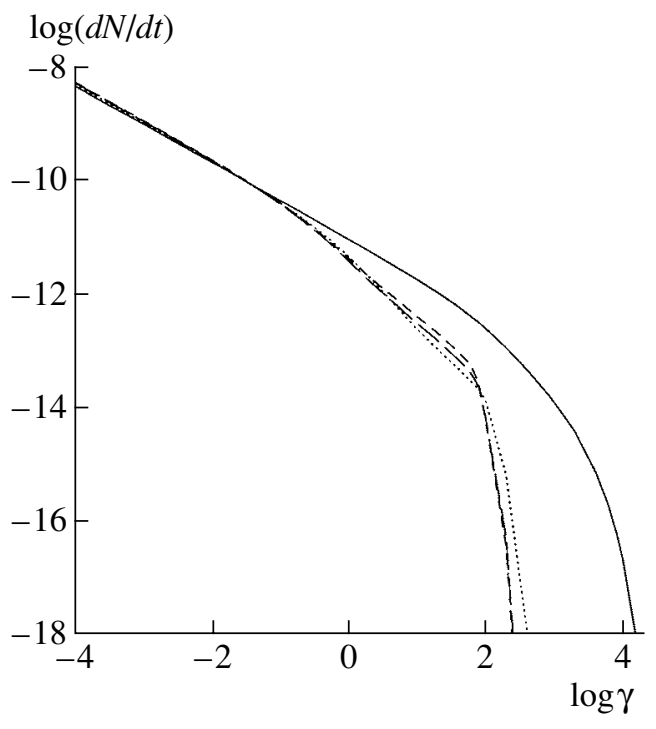

Fig. 2. Same as Fig. 1 for $B=0.05 B_{\mathrm{cr}}, P=0.1 \mathrm{~s}$.

We used the following approximate expression for the coefficient for the absorption of a photon accompanied by the creation of an electron-positron pair when the photon has traveled through a path of $1 \mathrm{~cm}[21]$ :

$$
\begin{gathered}
k(x, \gamma, \Psi)=\frac{3}{16} \sqrt{\frac{3}{2}} \alpha_{\mathrm{F}} \frac{1}{\lambda_{\mathrm{c}}} \\
\times \frac{B}{B_{\mathrm{cr}}} \sin \Psi \exp \left(-\frac{8}{3} \frac{B_{\mathrm{cr}}}{B \gamma \sin \Psi}\right),
\end{gathered}
$$

where $\quad B_{\mathrm{cr}}=m^{2} c^{3} / e \hbar=4.41 \times 10^{13} \mathrm{G}, \quad$ and $B=B(x)$ is the magnetic-field strength at the point $x$. Beginning from some height $\eta_{\mathrm{c}}$, the electron-positron plasma created by the absorption of the gamma-rays screens the parallel electric field and creates the upper diode plate (anode); i.e., when $\eta \geq \eta_{\mathrm{c}}$, the potential can be considered to be constant along the field lines: $\Phi(\eta, \xi, \phi)=\Phi\left(\eta_{\mathrm{c}}, \xi, \phi\right)$.

As $\eta_{\mathrm{c}}$, we take the height $\eta$ at which the growth coefficient $Q$ is equal to 0.1 on the field line ( $\xi=0.5$, $\phi=0)$. Similar values for $Q\left(\eta_{\mathrm{c}}\right)$ were adopted in $[4,11]$. Note that a very different choice of $Q$ (for example, $Q \approx 0.01$ ) leaves $\eta_{\mathrm{c}}$ virtually unchanged (for $\xi$ not very close to 0 and 1 ). This is associated with the rapid growth in $Q$ near $\eta_{\mathrm{c}}$ during the generation of electron-positron pairs by the curvature radiation.

The optical depth $\tau$ associated with the absorption of photons in the magnetic field (with the creation of electron-positron pairs) is taken to be

$$
\tau(\gamma, y, x)=\int_{\lambda_{y}}^{\lambda_{x}} k(z(\lambda), \gamma, \Psi(y, z(\lambda))) d l_{z},
$$




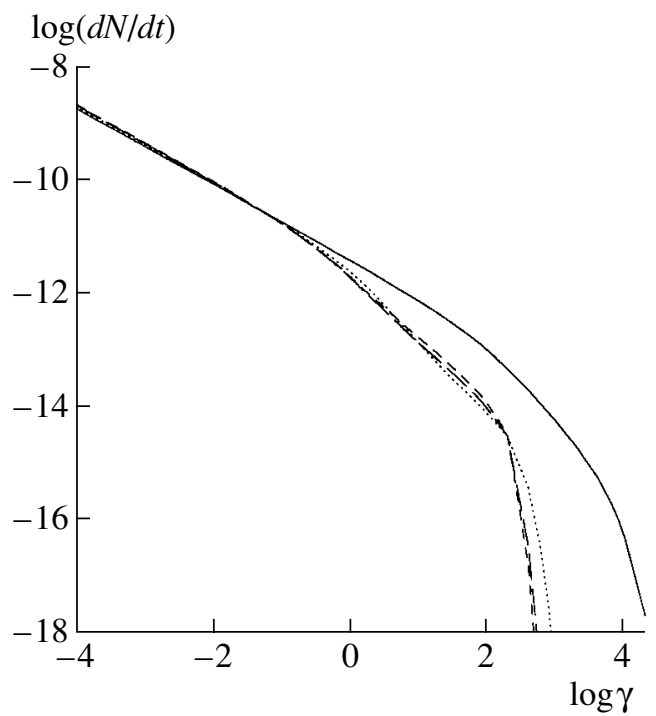

Fig. 3. Same as Fig. 1 for $B=0.02 B_{\mathrm{cr}}, P=0.1 \mathrm{~s}$.

where the integration is carried out over the photon path $z(\lambda)$ (taken to be a straight line) from the point $y$ to the point $x\left(z\left(\lambda_{y}\right)=y, z\left(\lambda_{x}\right)=x\right), \Psi(y, z)$ is the angle between the momentum of a photon emitted at the point $y$ and $\mathbf{B}(z)$ at the point $(y, z)$, $d l_{z}=\left|\frac{d \mathbf{z}}{d \lambda}\right| d \lambda$, and $\lambda$ is a parameter measuring distance along the curve. For photons with $\gamma \leq 2$, we assume $\tau \equiv 0$.

The intensity of the generation of electronpositron pairs is calculated as follows. We first construct the curve $y(\lambda)$, such that photons emitted from points $y(\lambda)$ pass through the point $x$, then calculate the integral

$$
\begin{gathered}
q_{+}(x)=\int d l_{y} \int_{2}^{+\infty} d \gamma K(x, y(\lambda)) n_{\mathrm{e}}(y(\lambda)) \\
\times k(x, \gamma, \Psi(y(\lambda), x)) \frac{d N}{d t}(y(\lambda), \gamma) \\
\quad \times \exp (-\tau(\gamma, y(\lambda), x)),
\end{gathered}
$$

where $q_{+}$is the number of positrons created at $x$ per unit volume per second, the integral $\int d l_{y}$ is taken along the entire curve $y(\lambda), d l_{y}=\left|\frac{d \mathbf{y}}{d \lambda}\right| d \lambda$, $n_{\mathrm{e}}(y)=|\rho(y) / e|$ is the number density of primary electrons at $y$ [calculated using (7)], and $K(x, y) \approx$ $\eta_{x}^{2} / \eta_{y}^{2}$ is a coefficient describing the decrease in the number density of photons as they propagate from the point where they were emitted.

We find the multiplication factor $Q$ at $x=$

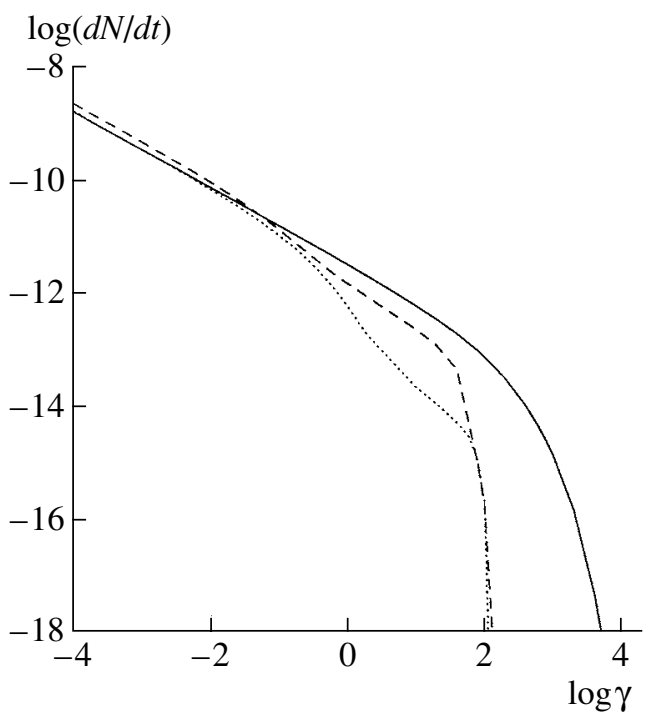

Fig. 4. Same as Fig. 1 for $B=0.05 B_{\mathrm{cr}}, P=0.2 \mathrm{~s}$, and an angle between the line of sight and the rotational axis of the pulsar equal to $7^{\circ}$.

$\left(\eta_{x}, \xi, \phi\right)$ using the expression

$$
Q(x)=\frac{1}{n_{\mathrm{e}}(x) c} \int_{1}^{\eta_{x}} \frac{B(x)}{B(y)} q_{+}(y)\left|\frac{d \mathbf{y}}{d \eta_{y}}\right| d \eta_{y},
$$

where $y=\left(\eta_{y}, \xi, \phi\right)$ and the integration is carried out along a magnetic field line.

The intensity of the radiation in the $\mathbf{n}$ direction is calculated as follows:

$$
\begin{aligned}
& \frac{d F}{d \Omega d \gamma}=\int d l_{y} K(y(\lambda)) \frac{d N}{d t d \gamma}(y(\lambda), \gamma) \\
& \quad \times n_{\mathrm{e}}(y(\lambda)) \exp (-\tau(\gamma, y(\lambda), x(y(\lambda)))),
\end{aligned}
$$

where $\frac{d F}{d \Omega d \gamma} d \gamma$ is the number of photons with energy $\gamma$ in the interval $d \gamma$ emitted by the pulsar tube in the direction $\mathbf{n}$ into a solid angle $d \Omega$ per second, the integral $\int d l_{y}$ is taken along the entire curve $y(\lambda)$, such that photons emitted from points $y(\lambda)$ have momenta that are parallel to $\mathbf{n}$, the integration is stopped when the curve $y(\lambda)$ intersects the sphere $S$ with radius $a \eta_{\mathrm{s}}$ (and with its center at the point $\eta=0$ ), and $x(y)$ is the point at which the photons emitted at $y$ intersect the sphere $S$. In the cases considered below, we use the value $\eta_{\mathrm{s}}=20$. The coefficient $K(y) \approx \frac{4}{9} a^{2} \eta_{y}^{2}$ describes the decrease in the number density of photons as they propagate from the star.

\section{RESULTS}

Figure 1 shows time-averaged spectra of the curvature radiation of the pulsar tube (for a radio 
Height of upper diode plates $z_{\mathrm{c}}$ and the potential $\Phi$ at $\left(\eta=\eta_{\mathrm{c}}, \xi=0\right)$ in units of $\Gamma=\frac{e \Phi}{m c^{2}}$

\begin{tabular}{|c|c|c|}
\hline$\nu$ & $z_{\mathrm{c}} / 10^{-2}$ & $\Gamma / 10^{6}$ \\
\hline \multicolumn{3}{|c|}{$B=0.05 B_{\mathrm{cr}}, P=0.2 \mathrm{~s}$} \\
\hline 0 & 11.8 & 28.6 \\
\hline 0.1 & 2.0 & 2.0 \\
\hline 0.2 & 1.34 & 1.35 \\
\hline 0.3 & 1.09 & 1.2 \\
\hline 0.5 & 0.93 & 1.55 \\
\hline \multicolumn{3}{|c|}{$B=0.05 B_{\mathrm{cr}}, P=0.1 \mathrm{~s}$} \\
\hline 0 & 5.6 & 25.6 \\
\hline 0.1 & 1.56 & 2.0 \\
\hline 0.2 & 1.11 & 1.5 \\
\hline 0.3 & 0.92 & 1.45 \\
\hline \multicolumn{3}{|c|}{$B=0.02 B_{\mathrm{cr}}, P=0.1 \mathrm{~s}$} \\
\hline 0 & 10.0 & 30.6 \\
\hline 0.1 & 2.34 & 2.75 \\
\hline 0.2 & 1.62 & 1.9 \\
\hline 0.3 & 1.32 & 1.75 \\
\hline
\end{tabular}

pulsar) for the case when $B=0.05 B_{\mathrm{cr}}, P=0.2 \mathrm{~s}$, $\chi=10^{\circ}, k=0.15, \Delta=0.1$, and the angle between the line of sight and the rotational axis of the pulsar is $10^{\circ}$ (i.e., at some time, the line of sight will be directed parallel to the axis of the main dipole $\mathbf{m}$ ). The curves correspond to various values of $\nu: \nu=0$ (dipolar field case) (solid), $\nu=0.1$ (dotted), $\nu=0.2$ (long-dashed), $\nu=0.3$ (short-dashed), and $\nu=0.5$ (dash-dotted).

Figure 2 shows analogous spectra for the case $B=0.05 B_{\mathrm{cr}}, P=0.1 \mathrm{~s}$, and Fig. 3 the same for the case $B=0.02 B_{\mathrm{cr}}, P=0.1 \mathrm{~s}$. In both cases, the angle between the line of sight and the rotational axis of the pulsar is $10^{\circ}$.

The table presents the values of $z_{\mathrm{c}}$ and the potential $\Phi$ at the point ( $\eta=\eta_{\mathrm{c}}, \xi=0$ ) (in units of $e \Phi / m c^{2}$ ) used to derive these spectra.

Figure 4 shows curvature-radiation spectra for the same parameters (a radio pulsar) as in Fig. 1 $\left(B=0.05 B_{\mathrm{cr}}, \quad P=0.2 \quad \mathrm{~s}, \quad \chi=10^{\circ}, \quad k=0.15\right.$, $\Delta=0.1$ ), but with the angle between the line of sight and the rotational axis of the pulsar taken to be $7^{\circ}$ (this makes it possible to observe the curvature radiation nearly from the "bottom" of the pulsar tube when $\nu=0.3$ ).
Figures 5-7 present the dependence of the intensity of the curvature radiation on the pulsar phase for this same case $\left(B=0.05 B_{\mathrm{cr}}, P=0.2 \mathrm{~s}, \chi=10^{\circ}\right.$, $k=0.15, \Delta=0.1$ ) and for various values of $\nu$ : a dipolar field, $\nu=0$ (solid), $\nu=0.1$ (dotted), and $\nu=0.3$ (dashed). The left-hand and right-hand plots correspond to angles between the line of sight and the rotational axis of the pulsar of $10^{\circ}$ (the phase-averaged spectrum shown in Fig. 1) and $7^{\circ}$ (the phaseaveraged spectrum shown in Fig. 4), respectively.

Figure 8 shows the dependence of the growth coefficient $Q$ on height $z$ along a line of force $(\xi=0.5$, $\phi=0)$ for the same case $\left(B=0.05 B_{\mathrm{cr}}, P=0.2 \mathrm{~s}\right.$, $\chi=10^{\circ}, k=0.15, \Delta=0.1$ ).

We can see from Figs. 1-3 that, in the cases considered, there is a sharp drop in the intensity of the curvature radiation at energies $\gamma>1$, even in the presence of only weak nondipolarity of the magnetic field.

The main contribution to this effect is made by the sharp decrease in the height of the upper diode plate $z_{\mathrm{c}}$ and the associated drop in the electrostatic potential $\Phi$. At energies $\gamma>2$, a significant, though not as great, contribution is made by the increase in the optical depth $\tau$ for photons emitted near the surface of the $\operatorname{star}(z \leq \Delta)$. The fact that the region with large force-line curvatures is relatively small $(z \leq \Delta)$ also plays a role.

It turns out that the intensity of the curvature radiation at energies $\gamma<0.1$ depends only weakly on the nondipolarity of the magnetic field, $\nu$. This is due to the fact that, when $\gamma \ll \gamma_{c}$, the intensity of the curvature radiation (13) is virtually independent of the energy of the primary electrons $\Gamma$ and depends only weakly on the field-line curvature $\rho_{\mathrm{c}}$.

We have used the potential (6) in all our calculations, as is fully justified in the cases listed above. In the case of a dipolar field, this potential coincides with the potential of [18] when $z_{\mathrm{c}} \ll 1$, which makes it possible to use it when $z_{\mathrm{c}} \sim \Delta, \theta_{\mathrm{s}} \sim \Delta$ as well. In the cases considered, in the presence of strong nondipolarity of the field, the condition $z_{\mathrm{c}} \ll \theta_{\mathrm{s}}$ will be satisfied so that the potential (6) is close to the potential (12) nearly along the entire tube, enabling us to use this potential when $\theta_{\mathrm{s}} \sim \Delta$ as well.

With regard to the assumptions we have made about the form of the absorption coefficient, we note that, for the selected parameters $\left(B<0.1 B_{\mathrm{cr}}\right.$ and so forth) and in intervals where the photons are near the absorption threshold, $\gamma \sin \Psi=2$, the absorption coefficient $k$ is small, and these intervals make only a modest contribution to the optical depth $\tau$ (if, of course, the optical depth itself is not negligibly small). In addition, the use of more precise formulas for the absorption coefficient (see, for example, [22]) 


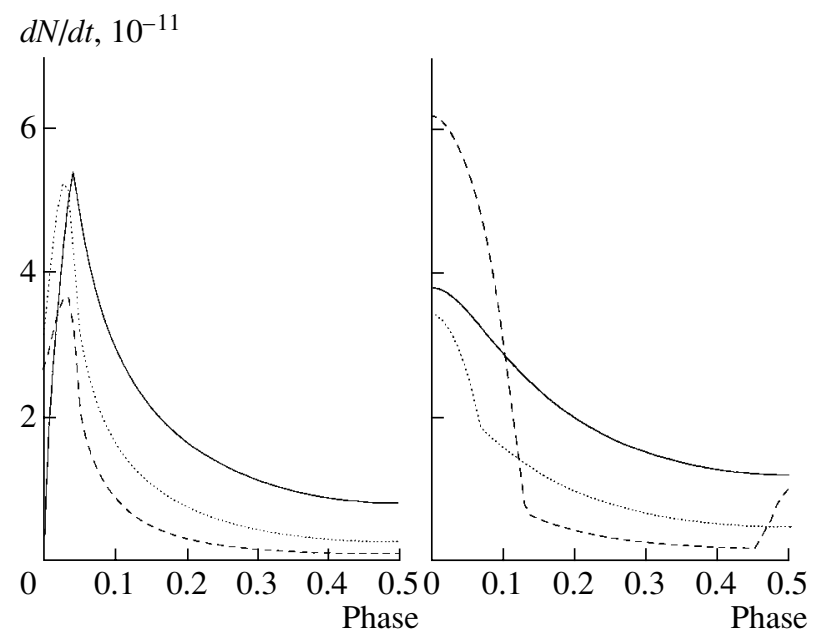

Fig. 5. Shape of the pulse of curvature radiation over half the pulsar period (the second half is symmetrical about the zero phase) for $B=0.05 B_{\mathrm{cr}}, P=0.2 \mathrm{~s}, \chi=10^{\circ}$, $k=0.15, \Delta=0.1$, and a photon energy of $100 \mathrm{keV}$. The angle between the line of sight and the rotational axis of the pulsar is $10^{\circ}$ in the left-hand plot and $7^{\circ}$ in the right-hand plot. The solid curve corresponds to a dipolar field $(\nu=0)$, and the dotted and dashed curves to nondipolar fields with $\nu=0.1$ and $\nu=0.3$, respectively. The intensity of the curvature radiation, $d N / d t$, is measured in units of the received flux from a source located at a distance of one parsec: photons $/ \mathrm{cm}^{2} \mathrm{~s} \mathrm{keV}$.

will likely lead to a decrease in its already small contribution to the optical depth in these intervals. Note as well that, with the selected parameters, the main contribution to the intensity of electron-positron pair creation $q_{+}$is made by photons located far from the threshold $\gamma \sin \Psi=2$ (apart from cases when $q_{+}$is very small and does not make a significant contribution to the growth coefficient $Q$ ).

Since we consider here radio pulsars without very strong magnetic fields $\left(B<0.1 B_{\mathrm{cr}}\right)$, we expect that the contributions of other processes (splitting of photons, absorption of photons with the formation of bound electron-positron pairs, and so forth) to the optical depth $\tau$ will be small (see, for example, [23]).

With regard to our disregard of general-relativistic effects, we acknowledge that, generally speaking, including these effects should change somewhat our final results. However, the main influence on the intensity and spectrum of the curvature radiation is exerted by the increase in the curvature of the magnetic field lines due to the curvature of space (i.e., compared to their value in a flat space; see, for example, [24]) and the curvature of the photon trajectories. Due to this latter effect, the photons will more rapidly achieve the angle $\Psi$ between their momentum and the magnetic field, which will lead to a growth in the optical depth $\tau$.

This means that we can formally consider the spectra presented above to be close to those that will

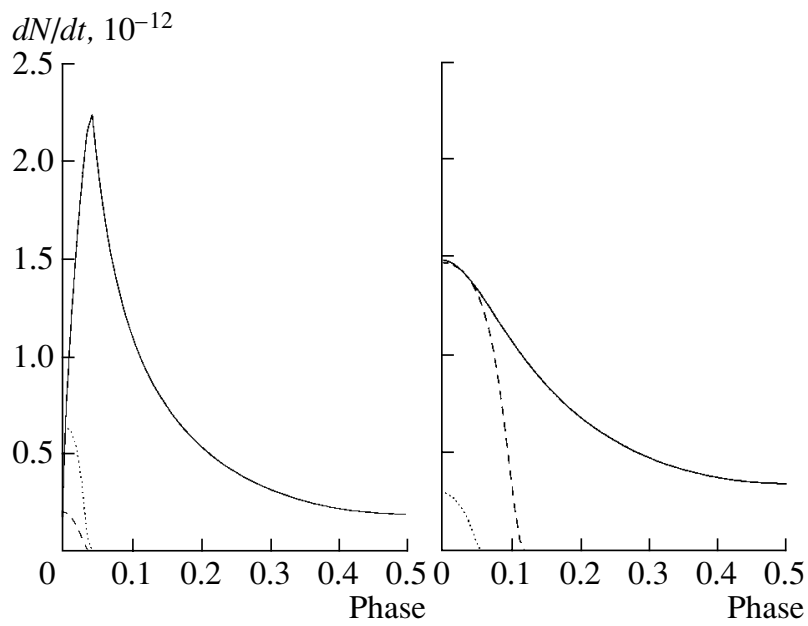

Fig. 6. Same as Fig. 5 for a photon energy of $10 \mathrm{MeV}$.

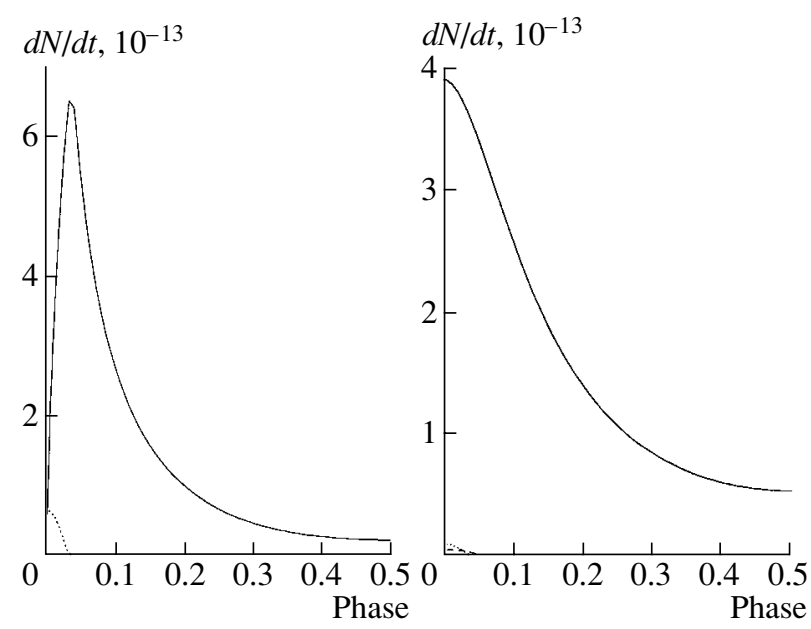

Fig. 7. Same as Fig. 5 for a photon energy of $50 \mathrm{MeV}$.

be obtained taking into account general-relativistic effects, but for weaker nondipolarity (possibly somewhat different from that considered by us here).

In connection with this, we expect that taking into account general-relativistic effects will only lead to a sharper drop in the intensity of the gamma-ray curvature radiation in the presence of small nondipolarity of the magnetic field, with the curvature $\mathrm{X}$-ray radiation being only weakly dependent on the nondipolarity of the magnetic field, as in the approximation we have considered here.

\section{CONCLUSIONS}

We have considered here the influence of asymmetry of the magnetic field on the X-ray and gammaray curvature radiation of radio pulsars, taking into account both the decrease in the radius of curvature of the magnetic field lines $\rho_{\mathrm{c}}$ and changes in the electric 


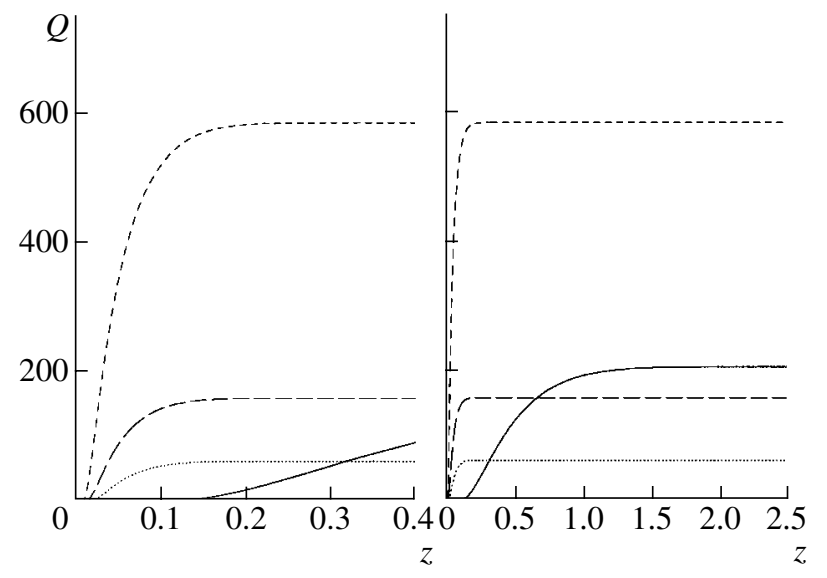

Fig. 8. Dependence of the multiplication factor for electron-positron pairs $Q$ on $z$ for $B=0.05 B_{\mathrm{cr}}$, $P=0.2$ s, $\chi=10^{\circ}, k=0.15, \Delta=0.1$ on the field line $(\xi=0.5, \phi=0)$. The solid curve corresponds to a dipolar field $(\nu=0)$, and the dotted, long-dashed, and shortdashed curves to nondipolar fields with $\nu=0.1, \nu=0.2$, and $\nu=0.3$, respectively.

field. As was shown in [13], the presence of a nondipolar component of the magnetic field can (in the case of a "favorable" configuration according to the criteria of Arons), other conditions being equal, appreciably increase the potential difference of the pulsar diode compared to its dipolar value; in particular, the potential (6) with $\nu=0.2-0.4$ can be a factor of two to three higher than its value for $\nu=0$.

In this "favorable" case, in the presence of not very strong nondipolarity of the magnetic field (beginning with $\nu=0.05-0.1$ and up to $\nu=0.5$ ), we observe a sharp decrease in the height of the upper diode plate $z_{\mathrm{c}}$ compared to its value for a dipolar field.

As a result, a sharp drop in the intensity of gamma-ray curvature radiation compared to the case of a purely dipolar magnetic field is observed, even in the presence of only weak nondipolarity $(\nu=0.05-0.1)$. At the same time, the intensity of the $\mathrm{X}$-ray curvature radiation $(\gamma<0.1)$ is affected only slightly.

In spite of the fact that we have investigated only one (rather approximate) model for including the effect of nondipolarity of the field on the intensity of the nonthermal $\mathrm{X}$-ray and gamma-ray radiation of radio pulsars, our results again demonstrate the need to take into account even weak (less than 10\%) nondipolarity of the magnetic field in calculations of the gamma-ray emission of the polar regions of radio pulsars.

\section{ACKNOWLEDGMENTS}

The authors thank V.D. Pal'shin and M.E. Gusakov for help with the numerical computations and for useful discussions. This work was supported by the Russian Foundation for Basic Research (project no. 04-02-17590) and the Program of Support for Leading Scientific Schools of the Russian Federation (grant no. NSh-1115.2003.2).

\section{REFERENCES}

1. M. D. Young, R. N. Manchester, and S. Johnston, Nature 400, 848 (1999).

2. J. A. Hibschman and J. Arons, Astrophys. J. 554, 624 (2001).

3. J. Gil and D. Mitra, Astrophys. J. 550, 383 (2001).

4. A. K. Harding and A. G. Muslimov, Astrophys. J. 556, 987 (2001); 568, 862 (2002).

5. A. K. Harding, A. G. Muslimov, and B. Zhang, Astrophys. J. 576, 366 (2002).

6. K. Hirotani, A. K. Harding, and S. Shibata, Astrophys. J. 591, 334 (2003).

7. L. Zhang, K. S. Cheng, Z. J. Jiang, and P. Leung, Astrophys. J. 604, 317 (2004).

8. A. G. Muslimov and A. K. Harding, Astrophys. J. 606, 1143 (2004).

9. M. A. Ruderman and P. G. Sutherland, Astrophys. J. 196, 51 (1975).

10. E. T. Scharlemann, J. Arons, and W. M. Fawley, Astrophys. J. 222, 297 (1978).

11. V. D. Pal'shin and A. I. Tsygan, Preprint No. 1718 (Ioffe Physicotechnical Institute, St. Petersburg, 1998).

12. A. I. Tsygan, in IAU Coll. No. 177: Pulsar Astronomy-2000 and Beyond, Ed. by M. Kramer, N. Wex, and R. Wielebinski (San Francisco, Astron. Soc. Pac., 2000); Astron. Soc. Pac. Conf. Ser. 202, $473(2000)$.

13. E. M. Kantor and A. I. Tsygan, Astron. Zh. 80, 665 (2003) [Astron. Rep. 47, 613 (2003)].

14. J. Gil and G. I. Melikidze, Astrophys. J. 577, 909 (2002).

15. U. Geppert, M. Rheinhardt, and J. Gil, Astron. Astrophys. 412, L33 (2003).

16. V. D. Pal'shin and A. I. Tsygan, Astron. Zh. 73, 426 (1996) [Astron. Rep. 40, 385 (1996)].

17. A. G. Muslimov and A. I. Tsygan, Mon. Not. R. Astron. Soc. 255, 61 (1992).

18. A. G. Muslimov and A. I. Tsygan, Astron. Zh. 67, 263 (1990) [Sov. Astron. 34, 133 (1990)].

19. V. S. Beskin, Pis'ma Astron. Zh. 16, 665 (1990) [Sov. Astron. Lett. 16, 286 (1990)].

20. L. D. Landau and E. M. Lifshitz, Course of Theoretical Physics, Vol. 2: The Classical Theory of Fields (Nauka, Moscow, 1988; Pergamon, Oxford, 1975).

21. T. Erber, Rev. Mod. Phys. 38, 626 (1966).

22. J. K. Daugherty and A. K. Harding, Astrophys. J. 273, 761 (1983).

23. A. E. Shabad and V. V. Usov, Astrophys. Space Sci. 102, 307 (1984).

24. E. Asseo and D. Khechinashvili, Mon. Not. R. Astron. Soc. 334,743 (2002).

Translated by D. Gabuzda 